\title{
Environmentally Friendly and Cheap Removal of Lead (II) and Zinc (II) from Wastewater with Fish Scales Waste Remains
}

\author{
Morlu G. F. Stevens ${ }^{1}$, Bareki S. Batlokwa ${ }^{1}$ \\ ${ }^{1}$ Botswana International University of Science and Technology, Department of Chemical and Forensic Sciences, Private \\ Bag 0016, Palapye, Botswana \\ Correspondence: Morlu G. F. Stevens, Botswana International University of Science and Technology, Department of \\ Chemical and Forensic Sciences, Private Bag 0016, Palapye, Botswana. E-mail: morlu.stevens@studentmail.biust.ac.bw
}

\author{
Received: May 22, 2017 Accepted: June 21, 2017 Online Published: August 8, 2017 \\ doi:10.5539/ijc.v9n4p22 URL: https://doi.org/10.5539/ijc.v9n4p22
}

\begin{abstract}
In this article, the physical and chemical properties of pulverized, vinegar treated waste from fish scale remains of fish from Lake Ngami in Sehitwa near Maun, Botswana, were investigated for a possibility of being employed as an environmentally friendly and cheap sorbent material for reducing or removing excess, toxic, heavy metal ions from wastewater before different uses. Lead (II) and Zinc (II) ions were selected as model ions to demonstrate the potential of fish scale waste remains in removing excess toxic heavy metal ions. The pulverized size of the waste was found to be 60 $\mu \mathrm{m}$, with round and smooth morphology, which are excellent characteristics usually associated with superior sorbents. Furthermore, the fourier transform infrared spectrometer spectrum showed multiple functional groups such as amines, carboxylic, hydroxyl, and carbonyls which are well known to bond well with metals through hydrogen and oxygen bonding. The X-ray diffractogram of the fish scales showed the presence of hydroxyapatite, which has an excellent ion-exchange performance, which exchanges calcium ion site with metals. Multivariate methodologies statistical software, Minitab, were employed for the simultaneous optimization factors that affect sorption studies; initial ions concentration which was found to be $24.45 \mathrm{mg} / \mathrm{L}$, the sorbents dose which was found to be $76.99 \mathrm{mg} / \mathrm{L}$, contact time, which were found to be $62.37 \mathrm{~min}$ and solution $\mathrm{pH}$ 7.52. The fish scales waste also exhibited high percentage removal efficiencies toward Lead (II) and Zinc (II) removal from real wastewater samples at $81.97 \%$ and $80.37 \%$ with percentage relative standard deviation of $1.34 \%$ and $1.02 \%$ respectively.
\end{abstract}

Keywords: environmentally friendly, fish waste, sorbents, heavy metals, and wastewater

\section{Introduction}

Freshwater scarcity has been one of the world's greatest concern over the last few decades. The advancement of technology, rapid pace of industrialization, population expansion, agricultural activities and unplanned urbanization have largely contributed to the severe shortage of freshwater. Furthermore, the small quantity of the available freshwater is constantly being polluted by among others, toxic metal ion containing; discharge of untreated sanitary and toxic industrial wastes, household effluent, and runoff from agricultural fields (Bhatnagar \& Sillanp, 2010). The pollutants are hazardous to the environment due to their toxicity and strong tendency to concentrate in the environment and in food chains (Agarwal \& Gupta, 2014).

Alternative water sources have now become a major focus for many countries, industries, companies and researchers (Renge, Khedkar, \& Pande, 2012). Reuse and recycling of water are currently employed as ways to curb the situation (Leflaive, 2009). Wastewater treatment has become one of the most widely used alternative water source for most countries around the world (Vigneswaran \& Sundaravadivel, 2004) but the removal of pollutants especially excess toxic heavy metal ions is costly and often employs toxic chemical to the environment. Wastewater containing excess, toxic heavy metal ions such Lead(II) and Zinc(II) causes detrimental effects to all forms of life upon direct discharge to the environment (Ahluwalia \& Goyal, 2007). In order to reduce the toxic heavy metal environmental pollution, a number of conventional physico-chemical removal methods, such as chemical precipitation (Fu \& Wang, 2011), electroplating (Hegazi, 2013), membrane separation (Rao, Kala, \& Daniel, 2006), evaporation (Paull \& Barron, 2004) and resin ionic exchange (Boyer \& Singer, 2005), have been employed remove the heavy metal ions from wastewater before use. These methods are expensive and non-environmentally friendly, thus cheaper and environmentally friendly alternative removal methods are sought after the world over.

It is for the above reasons that in this paper, we are employing, fish scales waste remains as an environmentally friendly 
and cheap method to simultaneously remove lead (II) and zinc (II). The study achieved the phenomenon of valuarizing waste material which is encouraged the world over.

\section{Method}

\subsection{Materials and Instrumentations}

The adsorbents used for the experiments were fish scales collected from fishes from Lake Ngami in Sehitwa near Maun, Botswana. Ultra-pure water of $18.0 \mathrm{M} \Omega / \mathrm{cm}$ resistivity, Type I, was prepared by a Elix 5 Millipore water purification system from Merck, (Darmstadt, Germany) and was used to prepare all solutions. Reagents used were: analytical grade $\mathrm{HCl}(37 \%)$ and $\mathrm{HNO}_{3}(60 \%)$ purchased from ACE (Johannesburg, South Africa), SPAR white spirit Vinegar, which was employed to treat the waste materials, was purchased from SPAR (Palapye, Botswana). Elemental standard solutions used (1000ppm $-\mathrm{Zn}$, and $\mathrm{Pb}$ ) and $\mathrm{NaOH}(97 \%)$ pellets were purchased from Rochelle Chemicals (Johannesburg, South Africa). A $0.45 \mu \mathrm{m}$ pore sized Whatman filter papers (Hardened Ashless Circles $45 \mathrm{~mm}$ ) that was used for all filtering processes were purchased from Sigma-Adrich (Johannesburg, South Africa). A Mars6 One Touch Macrowave Assisted Extractor/Digester (CEM Microwave Technology Ltd, North Carolina, USA) obtained from CEM (Johannesburg, South Africa) was employed to digestion and extraction of heavy metals from the fish scales. For determination of size, morphology and nanoparticle composition, JSM 1700 SEM coupled with EDX, obtained from (California, USA) was employed. Perkin Elmer System, Spectrum two Fourier transform infrared (FTIR) spectroscopy was employed to determine the functional groups of the fish scales. A powder D8 Advanced Powder X-Ray Diffractometer (XRD) obtained from Bruker (Karlsruhe, Germany) was employed for characterization of the fish scales. Heavy metals were determined by an iCAP 7000 series Thermo Scientific inductively coupled plasma spectrometer (ICP - OES), (Johannesburg, South Africa).

\subsection{Fish Scales Waste Pre-treatment}

The fish scales collected from fishes from Lake Ngami in Sehithwwa near Maun, Botswana. They were then washed thoroughly with deionized water to remove mud and other impurities. Then it was sun dried for 48 hours and pulverized employing a Fritsch pulverisette 5 pulveriser obtained from Fritsch (Berlin, Germany), operated at $400 \mathrm{rpm}$ for $90 \mathrm{~min}$ in both milling and reverse mode. The pulverized materials were then sieved to $63-200$-micron mesh size. After screening, they were again washed with deionized water several times to remove color and dust. The material was than treated with SPAR white spirit vinegar to remove inorganic pollutants. Finally, they were dried in an oven at $65 \pm 5{ }^{\circ} \mathrm{C}$ for 6 hours (Bhatnagar \& Sillanp, 2010).

\subsection{Fourier Transform Infrared Spectroscopy (FT-IR)}

Fourier transform infrared (FTIR) spectrometer was used to identify the functional groups responsible for the heavy metals uptake and bonding possessed on the surface of the fish scales. The FTIR spectra were recorded in the wavelength range $500-4000 \mathrm{~cm}^{-1}$ on a Nicolet iS10 Thermo Scientific FTIR. The data were collected at $2.0 \mathrm{~cm}^{-1}$ resolution, and each spectrum was a result of 256 scans.

\subsection{X-ray Powder Diffraction (XRD)}

A powder D8 Advanced Powder X-Ray Diffractometer (XRD) analysis was employed to investigate the physical properties as it relates to the crystallinity of the waste material. The XRD was operated with $\mathrm{Cu} \mathrm{K} \alpha$ emission $(\chi=$ $1.54105 \AA$, $40 \mathrm{kV}, 40 \mathrm{~mA}$ per sec) and with high efficiency linear detector of Lynx Eye type. The scanning mode used was coupled with $2 \Theta / \Theta$ on the scanning range $10^{\circ}-120^{\circ}$ values. The crystallite size of the sample was calculated by Deby-Scherrer method.

\subsection{Scanning Electron Microscopy Coupled with Energy Dispersive X-ray Spectroscopy (SEM-EDX)}

Scanning Electron Microscope coupled with Energy-dispersive X-ray spectrometer (SEM - EDX) (JSM - 7100F), was employed to determine the surface morphology of the fish scales and to determine its elemental composition. An E6700 Polaron range high vacuum pressure sputter coater (Quorum Technologies, UK) was employed to coat the fish scales with carbon. These were then taken for SEM-EDX analysis, which operated under high vacuum and beam acceleration voltage of $10.0 \mathrm{kV}$ (the recommended operating voltage for organic material samples). The results from this analysis were then used to determine the surface morphology and elemental composition of the fish scales.

\subsection{Determination of Heavy Metals within the Fish Scales Waste}

$3 \mathrm{~g}$ of the fish scales was weighed and then digested employing a MARS6 microwave assisted digester in an aqua regia solution of $\mathrm{HCl}: \mathrm{HNO}_{3}$ at a ratio of $3: 1 \mathrm{v} / \mathrm{v}$. The mixture was placed in $100 \mathrm{~mL}$ TFM sample vessel and digested at a pressure of $600 \mathrm{psi}$, temperature of $100{ }^{\circ} \mathrm{C}$ and $1200 \mathrm{~W}$. The ramp time was set at $20.0 \mathrm{~min}$ with a hold time of $10.0 \mathrm{~min}$. The resulting volume was filtered using a whatman No. 1 filter paper and put into $50 \mathrm{~mL}$ volumetric flasks. Deionized water was added to the flasks and filled up to the mark. The samples were investigated for heavy metals employing ICP-OES. 


\subsection{Batch Adsorption Studies for Zn (II) and Pb (II) Removal Employing Fish Scales Waste}

All experiments were carried out in batches and done in triplicates. A $100 \mathrm{mg} / \mathrm{L}$ standard mixture of $\mathrm{Zn}$ (II) and $\mathrm{Pb}$ (II) was prepared from $1000 \mathrm{ppm}$ stock solution of each of the metals. The fish scales employed were of particle size of $\leq 63 \mu \mathrm{m}$. A multivariate optimization methodology employing Minitab Release 14 statistical software (Minitab Inc., USA).

\subsection{Optimization of Adsorptive Parameters of the Fish Scales Waste}

Optimization studies were carried out using multivariate optimization methodology. In this study, the fish scales were optimized by looking at four factors, namely contact time, $\mathrm{pH}$, sorbent dosage, as well as initial concentration. These were first screened through the use of a two-level fractional factorial design. This enabled identification of the significance of each factor towards the experimental output. Following this, a face centered central composite design was then performed to determine the optimum conditions for each factor that would result in a maximized response of the experiments. The optimization process was carried out with the use of Minitab Release 14 statistical software (Minitab Inc., USA).

The screening design was carried using the experimental conditions as described in Table 1 . The effluent was filtered into a $50 \mathrm{~mL}$ volumetric flask and deionized water added to the mark. It was investigated for the selected metal ions employing ICP-OES. The experiments were done in triplicates to evaluate the adequacy of the method and lack-of-fit.

Table 1. Factors and their levels for the two-level fractional factorial design for the optimization of the eggshell biomass

\begin{tabular}{llll}
\hline Variable & Factor & Low level & High level \\
\hline A & dosage $(\mathrm{mg})$ & 25 & 1000 \\
B & $\mathrm{pH}$ & 2 & 10 \\
C & Contact time (minutes) & 15 & 90 \\
D & Concentration $(\mathrm{mg} / \mathrm{L})$ & 0 & 50 \\
\hline
\end{tabular}

After the factorial screening, a central composite design (CCD) was used as the experimental matrix for the response surface method. The significant factors from the screening phase (two-level $1 / 2$ fraction factorial design) were all used for the optimization phase (CCD). Two replicates were also carried out for this set of experiments. It was then filtered into a $50 \mathrm{~mL}$ volumetric flask and deionized water added to the mark and the effluent investigated for metal ions employing ICP-OES.

\subsection{Application of the Optimized Fish Scales Waste Adsorption Method to Real Samples}

The extraction efficiency for the fish scales was investigated by applying the optimized parameters to wastewater samples collected from Gaborone waste water treatment plant. The fish scales were used for $\mathrm{Zn}^{2+}$ and $\mathrm{Pb}^{2+}$ removal in the samples. $50 \mathrm{~mL}$ of water samples were used and the optimized conditions applied to the water samples. The mixture was subjected to a rotary shaker at $200 \mathrm{rpm}$ for the optimized time, after which it was then filtered using a whatman No. 1 filter paper and put into $50 \mathrm{~mL}$ volumetric flasks. Deionized water was added to the flasks and filled up to the mark. The analysis was done in triplicates. $\mathrm{Zn}^{2+}$ and $\mathrm{Pb}^{2+}$ standards (from $5 \mathrm{ppm}$ to $50 \mathrm{ppm}$ ) were prepared for the calibration curve. The analytes remaining in the water sample after adsorption were determined with ICP - OES and the results analysed using Microsoft Excel 2016.

\section{Results and Discussions}

\subsection{Preparation of Fish Scales Waste Remains Prior to Application}

The pulverized fish scales were sieve to a particle size of $\leq 63$ microns. A $750 \mathrm{ml}$ of SPAR white spirit vinegar consisting of $12.67 \%$ acetic acid, was employed as a treatment reagent to remove inorganic substances that were present within the fish scales. This was done to avoid further pollution or infestation of the wastewater sample once the fish scales (adsorbent) are being applied for adsorption of the selected metals. Characterization of the fish scales showed the effectiveness of the vinegar during the treatment process. Figure 1 show the treated fish scales which reflected a whitish-gray colour. 


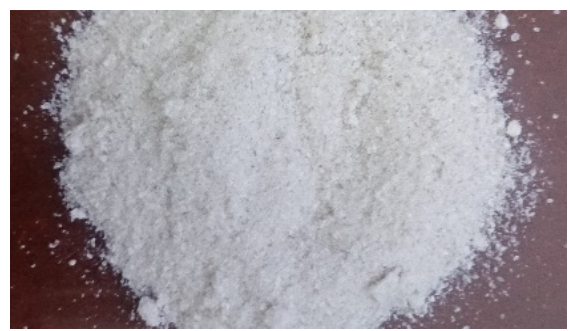

Figure 1. Prepared fish scales powders

\subsection{Fourier Transform Infrared Spectroscopy (FT-IR)}

Fourier Transform Infrared Spectrometer (FTIR) was used to determine the functional groups responsible for analyte interaction with the sorbents. Figure 2 below shows the functional groups of fish scales before removal (black) and after removal (red) of inorganic ions. The functional groups that are prominent in the fish scales are $1016 \mathrm{~cm}^{-1}$ due to carboxyl bands and primary amines, 554 to $597 \mathrm{~cm}^{-1}$ due to alkanes, $870 \mathrm{~cm}^{-1}$ due to sulphonates, $-\mathrm{OH}$ and N-H groups at $3286 \mathrm{~cm}^{-1}$, C-O group at 1542.1 to $1642.4 \mathrm{~cm}^{-1}$ and $\mathrm{C}-\mathrm{H},-\mathrm{CH}_{3},-\mathrm{CH}_{2}$ groups at $1408.4 \mathrm{~cm}^{-1}$

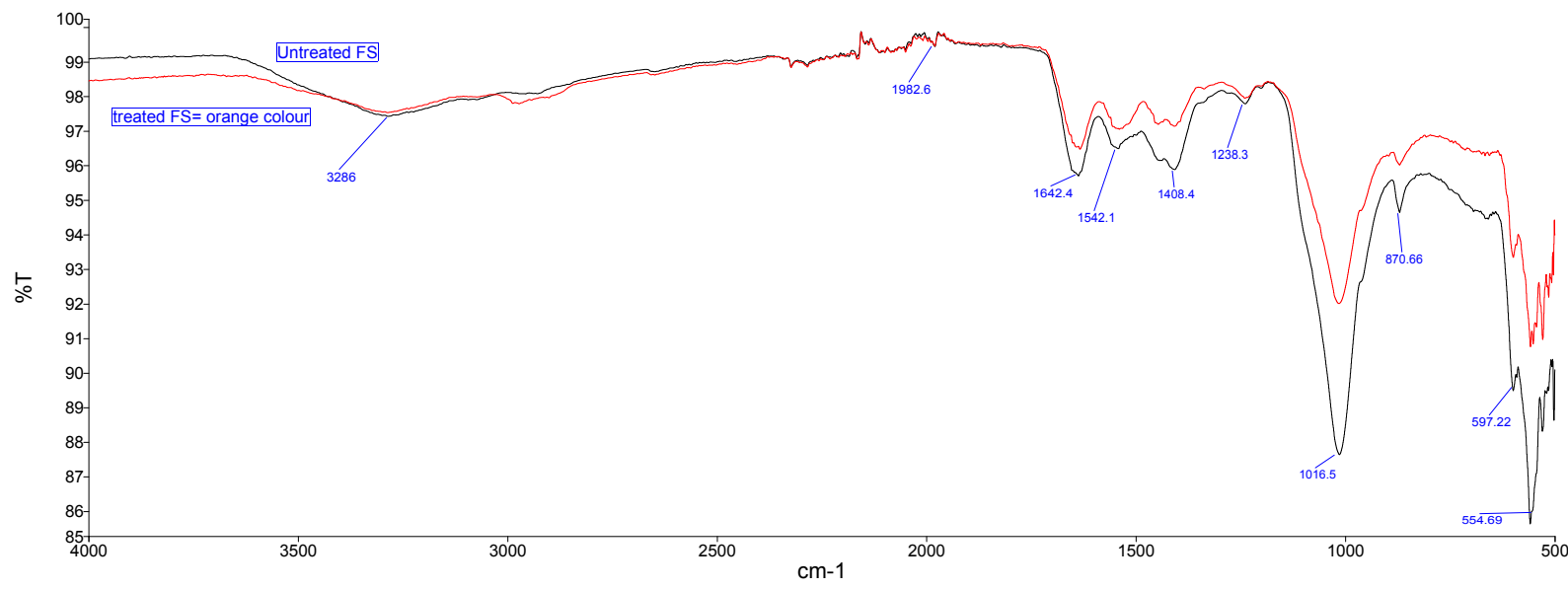

Figure 2. FTIR of fish scales before removal (black) and after removal (red) of inorganic ions

The heavy metals interact with different functional groups and cause the decrease of intensities and shift of the mentioned peaks as shown in Figure 2. These interactions could be due to complex formation of analytes with the surface functional groups. Complexation of the metal ions with the different functional groups is responsible for the removal of metal from the water samples. Metal ion forms coordinate bonds with the different functional groups and hence cause a decrease in peak intensity and also shift in peaks. Altering $\mathrm{pH}$ helps in changing the surface charge of the sorbents thereby improving on the removal efficiency of the sorbent. The functional groups such as amines, carboxylic, hydroxyl, carbonyls form anionic sites as $\mathrm{pH}$ is raised from acidic to basic conditions.

\subsection{X-ray Powder Diffraction (XRD)}

The XRD phase analysis of the fish scales were performed using JCPDS (Joint Committee on Powder Diffraction Standards) card number 01-073-0293 that showed a compound of hydroxyapatite with a chemical formula $\mathrm{Ca}_{5}\left(\mathrm{PO}_{4}\right)_{3}(\mathrm{OH})$, and $\mathrm{Ca}_{10}\left(\mathrm{PO}_{4}\right)_{6}(\mathrm{OH})_{2}$ for the treated fish scales before application (TFs), and the fish scales powder after adsorption (AFs) respectively (see figure 3 ). The powder X-ray diffraction patterns of the fish scales (TFs and AFs) showed increasing intensity of the reflections in the order of AFs $>$ TFs, with $d$-spacings of $0 \cdot 735,0 \cdot 564,0 \cdot 534,0 \cdot 466$, $0 \cdot 401,0 \cdot 342,0 \cdot 243$, and $0 \cdot 182$ for AFs, and $0 \cdot 242$ and $0 \cdot 189$ for TFs corresponding to the hydroxyapatite structure (Kumari \& Rath, 2014). 


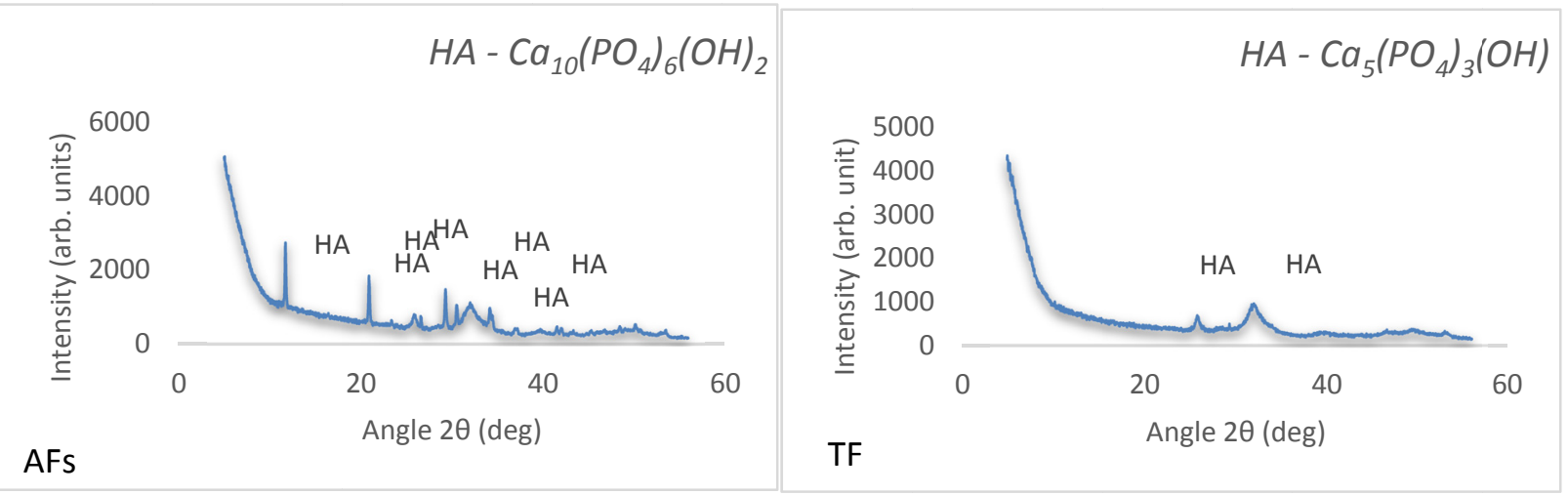

Figure 3. X-ray diffractogram of the fish scales before removal (TFs) and after removal (AFs)

\subsection{Scanning Electron Microscopy Coupled with Energy Dispersive X-ray Spectroscopy (SEM-EDX)}

Figure 4 shows SEM micrographs of the fish scales powders. The treated fish scale (TFs) is shown in Fig. 4B. The fish scale appears to have a rough surface and are characterized by having two regions, one being darker and the other being white. The white region is rich in inorganic material containing high proportion of calcium and phosphorus whereas the dark region is rich in protein because it has high proportion of carbon and oxygen. Fig. 4A and 4B represents the micrograph of the treated fish scales (TFs) and of the heavy metals loaded fish scale (AFs). Figure 4 clearly shows that the presence of new shiny bulky particles over the surface of ions loaded fish scale (AFs) which are absent in the TFs. These results confirm the binding of metals in fish scale through adsorption.

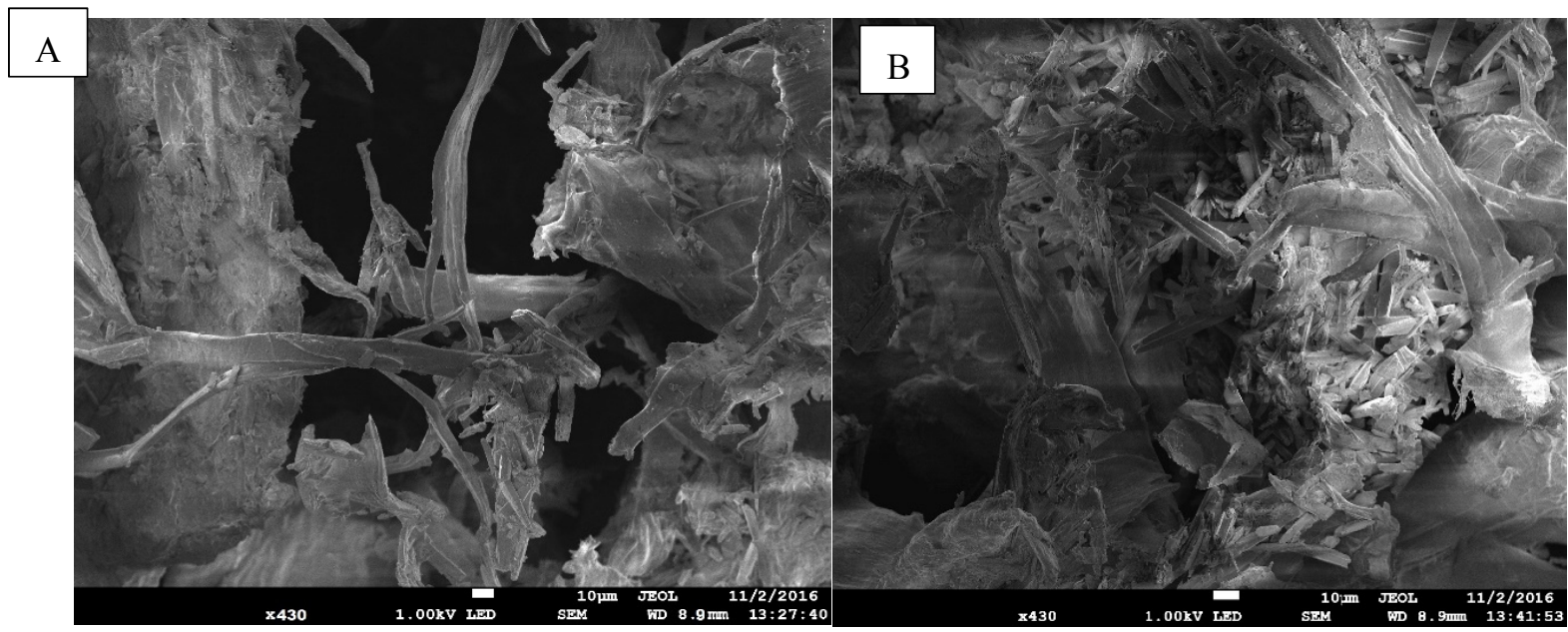

Figure 4. SEM images of the fish scales Waste A) before removal (TFs) and B) after removal (AFs)

\subsection{Determination of Heavy Metals within the Fish Scales}

The concentration of $\mathrm{Zn}$ (II) and $\mathrm{Pb}$ (II) in the untreated fish scales and treated fish scales were determined from an external calibration curve obtained using standards of known concentration. It was observed that $\mathrm{Pb}^{2+}$ was not detected with in the untreated fish scale, however, $\mathrm{Zn}^{2+}$ was detected at a concentration of $0.013 \mathrm{mg} / \mathrm{g}$. After treatment with the vinegar, $\mathrm{Zn}^{2+}$, that were present within the untreated fish scales was no longer detectable. All the concentrations were within the recommended limits for edible food set by FAO 2012 and WHO 1999.

\subsection{Optimization of Adsorptive Parameters of the Fish Scales}

Experimental matrices were designed using Minitab for the optimization purposes. Optimization was carried out for adsorption efficiency of each of the fish scales. The responses were followed by the use of ICP - OES absorbance measurements for the heavy metals.

Prior to performing the actual optimization, a $1 / 2$ fraction factorial design was carried out in order to assess the level of significance of each factor investigated for. The factorial design comes as a screening phase; screening designs allow screening a relatively large number of factors in a relatively small number of experiments that cover the whole experimental domain, with the output identifying the most influential factors towards obtained yields. Analysis of this data was in the forms of normal probability plots of standardized effects, Pareto charts of standardized effects and 
residuals plots; as shown by Figures 5, 6 and 7, respectively. From the normal probability plot of standardized effects (Figure 5), the magnitude of the main effects of each factor as well as the effects brought about by the interaction of factors, towards the obtained yield are investigated. The magnitude of each type of effect is represented by its distance from the solid line, as well as the side on which the effect lies with respect to the solid line. Negative effects lie to the left while positive effects lie to the right of the solid line. The solid line indicates where the points would fall if the effects were zero, while the percentage in the y-axis signifies the weightage of each factor's contribution towards the obtained yield. All the investigated effects showed a positive magnitude as they all lay to the right of the graph, as can be observed in Figures 5. The main effects, due to Factors A (Contact time), B (pH), C (sorbent dosage) and D (Concentration) were significant for the selected ions. Figures 5 show that the Factor D for the fish scales powders lay furthest to the right of the solid line, signifying that factor $\mathrm{D}$ had a greater positive magnitude towards contribution of the yields obtained. Meanwhile, the contribution of individual factors (A, B, C, and D) showed higher weightage towards the output as compared to that of effects brought about by the interaction of factors for the fish scales. The fish scales powder normal probability plots of standardized effects of $\mathrm{Zn}^{2+}$ showed that there was a significant contribution towards the obtained yield as a result of interaction between factors (Factor A and Factor C).
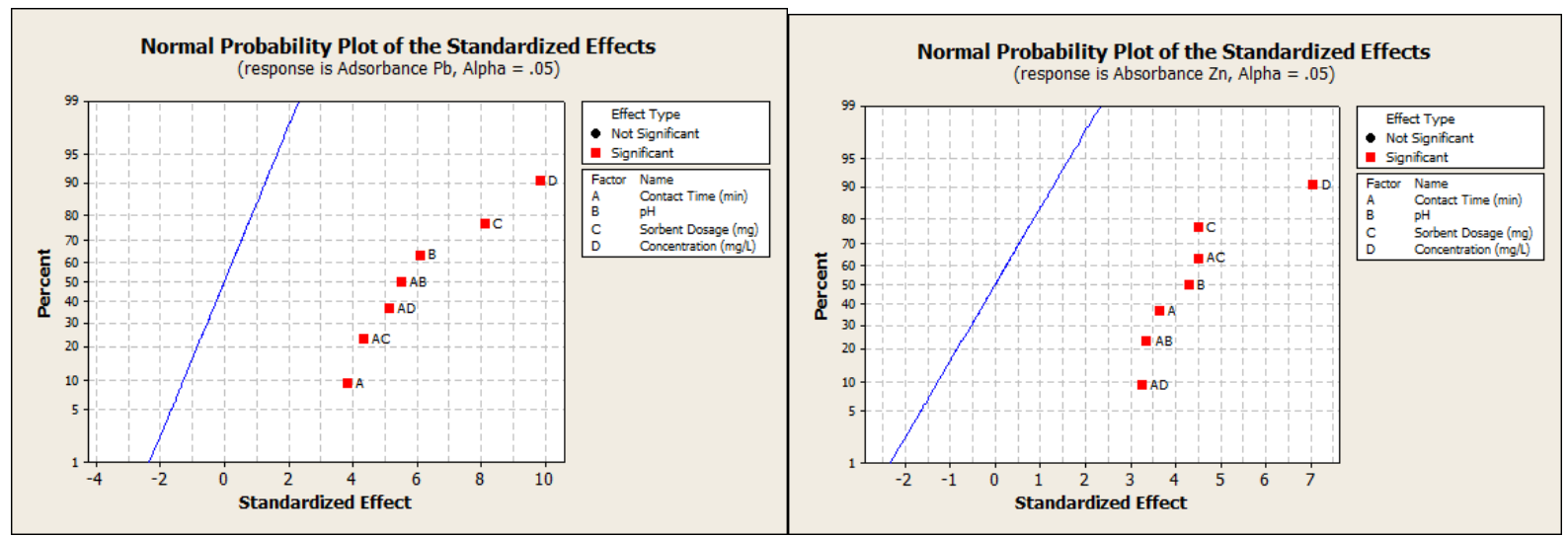

Figure 5. The normal probability plots of standardized effects of ions for the fish scales

Pareto chart of standardized effects (Figure 6) is used for the identification of factors and interaction effects most significant in the study. Only the absolute values of the effects are displayed. A reference line is drawn on the graph, above which any effect that crosses it is most significant. The reference line is calculated by Minitab based on standardized effects (if an error term exists) or through the use of Lenth's pseudo-standard error (if no error term is present) (Prakash, Talat, Hasan, \& Pandey, 2008). In simple terms, this chart can be thought of as application of a p-value test at a $95 \%$ confidence level. The Pareto chart displays statistically relevant effect of each factor on the response, sorted from largest to smallest; with the reference line corresponding to a p-value of 0.05 (Ponnusami, Madhuram, Krithika, \& Srivastava, 2008). Interpretation of this graph leads to the same conclusions drawn as when using the normal probability plot of standardized effects. For Figures 6, the main effects [due to Factors A (contact time), $\mathrm{B}(\mathrm{pH}), \mathrm{C}$ (sorbent dosage) and D (concentration) were all significant as each term crossed the reference line as well as factors interactions. The effect of Factor D showed the highest significance towards adsorption efficiency of fish scales.
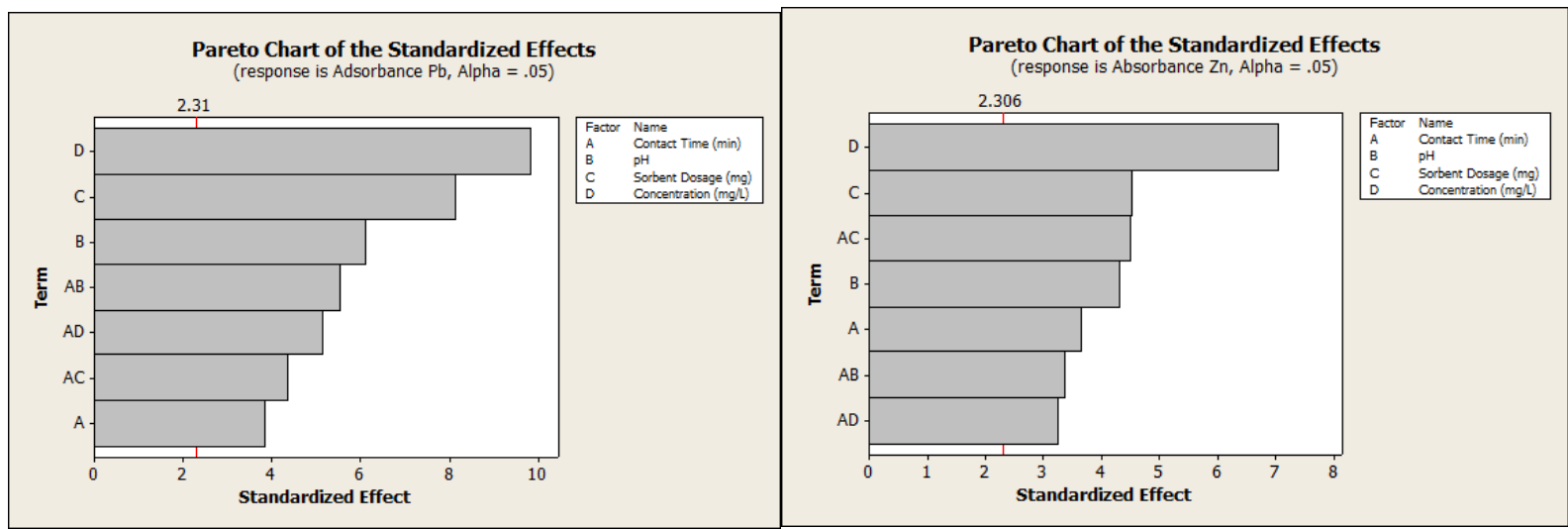

Figure 6. Pareto charts of standardized effects on fish scales

Figure 7 on the other hand, shows the residual plots for the yield (absorbance) obtained when using the fish scales. The plots probe into the distribution pattern of data points through the use of residuals. Residuals are the result of the 
difference between the observed and the fitted values (Pokhrel \& Viraraghavan, 2006). Normal probability and histogram plots investigate whether the data obtained exhibits a standard Gaussian distribution. For normal probability plots, if the data points fall approximately along the straight line, then the residuals are said to be normally distributed, meaning the data follows the Gaussian distribution (Mathews, 2004). This was the observed behavior as shown in Figure 7. A plot of residuals against fitted responses/values is used to detect unequal error variances and outliers, while the plot of residuals against order of the data checks for correlation of the residuals. The residuals against fitted values plot revealed a constant variance of the residuals about the center line. The plot of residuals against order of the data showed a randomized fluctuating pattern about the center line, signifying that the data was uncorrelated with each other. All the plots for the fish scales show that the residuals were randomly distributed, thus signifying absence of systematic errors and hence adequacy of the model.
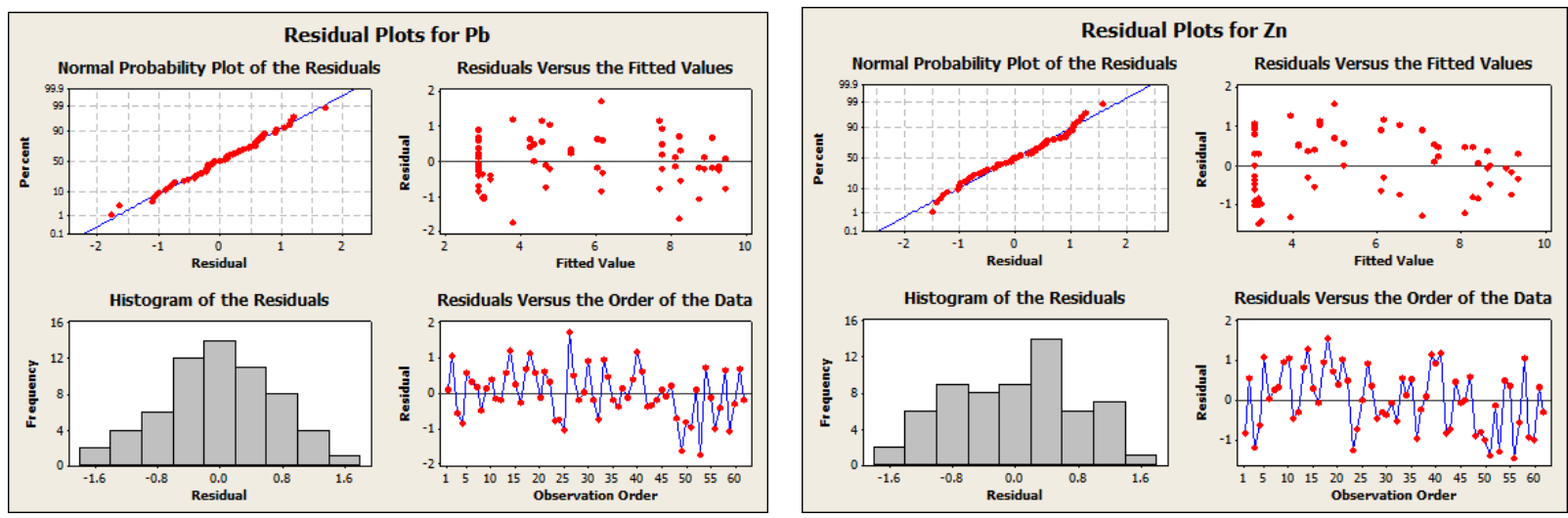

Figure 7. Residuals plots of standardized effects on the fish scales

The statistical significance of the model applied was evaluated through the use of Fisher distribution (F-test) for analysis of variance (ANOVA) to validate the linear model at $95 \%$ confidence level. Furthermore, the regression coefficient, $R^{2}$, was also used to assess the fit of the model to the experimental data. These are shown by Table 2 and Table 3, of which the values were found to be $94.1 \%\left(\mathrm{~Pb}^{2+}\right)$, and $99.5 \%\left(\mathrm{Zn}^{2+}\right)$. The lack-of-fit test was also used to further examine the adequacy of the model. Lack-of-fit (LOF) is a check for the applied model, to assess if the error arising from contribution by regressor-response relationship is accounted for. The p-value of the LOF test must be less than the significance level $(\alpha)$ for there to be an insignificant LOF (Ponnusami, Krithika, Madhuram, \& Srivastava, 2007). An insignificant LOF implies that the model accounts for the errors brought about by the regressor-response relationship. In this work, $\alpha$ was 0.05 for the metals for the fish scales. The p-value for the LOF was found to be 0.000 for the metals. This signifies adequacy of the model in that there is no LOF shown by the applied model.

Table 2. Analysis of Variance for $\mathrm{Pb}$

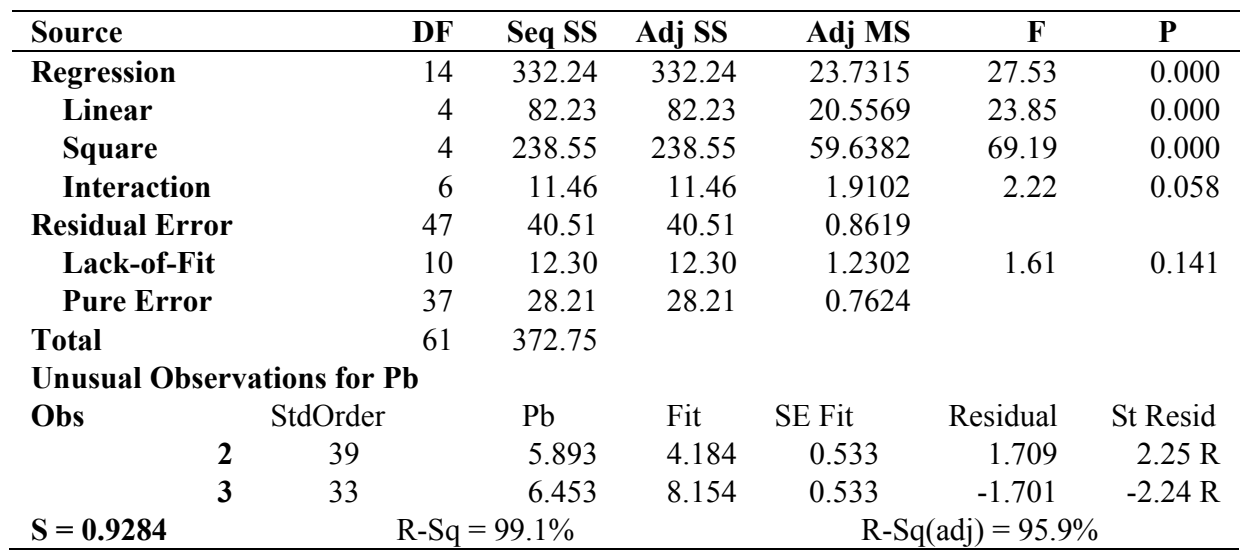


Table 3. Analysis of Variance for $\mathrm{Zn}$

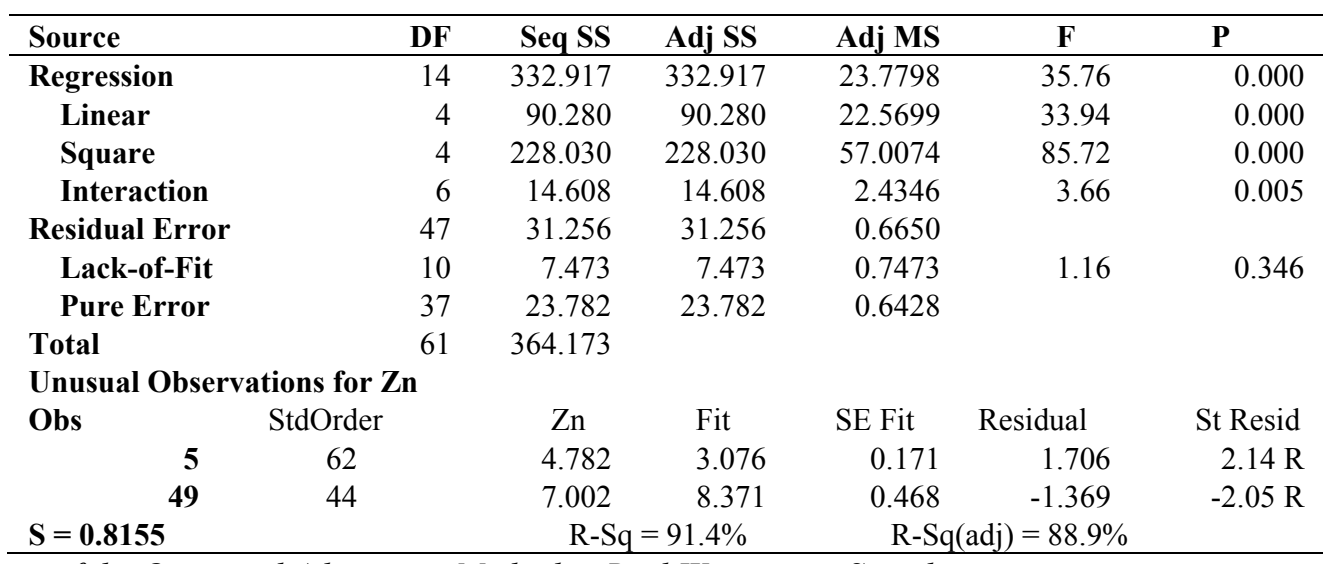

\subsection{Application of the Optimized Adsorption Method to Real Wastewater Samples}

Prior to application of the method to wastewater sample, the wastewater sample was spiked with $2 \mathrm{mg} / \mathrm{L}$ of the each of the selected ions. The recoveries were found to be $102 \pm 2.03 \%$ and $107 \pm 1.44 \%$ for lead (II) and zinc (II) respectively. The obtained optimum parameters as shown in Table 4, were applied to a $50 \mathrm{~mL}$ of the wastewater sample solution in similar procedure as previously described. The percentage removal of the selected metals was calculated using the formula below

$$
\frac{C i-C f}{C i} \times 100
$$

Equation 1

Where

$\mathrm{Ci}$ is the initial concentration of metal ions in wastewater sample

$\mathrm{Cf}$ is the final concentration of metal ion in wastewater after applying the fish scales

The percentage removal of $\mathrm{Zn}^{2+}$ and $\mathrm{Pb}^{2+}$ were $81.97 \pm 1.023$ and $80.37 \pm 1.45$ at $\mathrm{n}=3$ respectively.

Table 3. Optimal parameters for adsorptive efficiency of the fish scales

\begin{tabular}{lllll}
\hline pH & Sorbent Dosages (mg) & Contact Time (min) & $\begin{array}{l}\text { Initial } \\
(\mathbf{m g} / \mathbf{L})\end{array}$ & Concentration \\
\hline $\mathbf{7 . 5 2}$ & 76.99 & 62.37 & 24.45 & \\
\hline
\end{tabular}

The treated fish scales showed a higher percentage removal towards $\mathrm{Zn}^{2+}$ then $\mathrm{Pb}^{2+}$, which could be attributed to either more interaction of $\mathrm{Zn}^{2+}$ to the functional groups present in the fish scales or to the fish scales' affinity for $\mathrm{Zn}^{2+}$ as compare to $\mathrm{Pb}^{2+}$.

\section{Conclusion}

This study indicated that fish scales which is widely available at low cost, can be used as an efficient adsorbent material for the removal of $\mathrm{Zn}^{2+}$, and $\mathrm{Pb}^{2+}$ from wastewater. IR spectrum analysis suggested the different functional groups which are present in the given samples are $\mathrm{OH}, \mathrm{CH}$ stretching, $\mathrm{C}=\mathrm{C}$ stretching, $\mathrm{C}-\mathrm{O}$ stretching. The $\mathrm{XRD}$ diffractogram suggested the presence of hydroxyapatite in the valuarized fish scales. Concentration of adsorbate, $\mathrm{pH}$, contact time, adsorbent dosage and adsorbent characteristics are the factors responsible for ions adsorption capability. This work demonstrated that fish scales waste remains are environmentally friendly, economical and readily available waste materials with high efficacy for the removal of excess toxic heavy metal ions from wastewater as shown by the by the two model ions, $\mathrm{Zn}^{2+}$ and $\mathrm{Pb}^{2+}$. Most importantly, this study has created an alternative method for agricultural and industrial waste management, particularly for fisheries and food industries. Furthermore, the study achieved the phenomenon of valuarizing waste material which is encouraged the world over.

\section{Acknowledgments}

This to acknowledge the Botswana International University of Science and Technology (BIUST) for fully funding this research.

\section{Conflicts of Interest}

The authors declare no conflict of interest. The founding sponsors had no role in the design of the study; in the collection, analyses, or interpretation of data; in the writing of the manuscript, and in the decision to publish the results. 


\section{References}

Agarwal, A., \& Gupta, P. K. (2014). Removal of Cu \& Fe from aqueous solution by using eggshell powder as low cost adsorbent. Advances in Applied Science Research, 5(2), 75-79.

Ahluwalia, S. S., \& Goyal, D. (2007). Microbial and plant derived biomass for removal of heavy metals from wastewater. Bioresource Technology, 98(12), 2243-2257. https://doi.org/10.1016/j.biortech.2005.12.006

Bhatnagar, A., \& Sillanp, M. (2010). Utilization of agro-industrial and municipal waste materials as potential adsorbents for water treatment-A review. Chemical Engineering Journal, 157(2-3), 277-296. https://doi.org/10.1016/j.cej.2010.01.007

Boyer, T. H., \& Singer, P. C. (2005). Bench-scale testing of a magnetic ion exchange resin for removal of disinfection by-product precursors. Water Research, 39, 1265-1276. https://doi.org/10.1016/j.watres.2005.01.002

Fu, F., \& Wang, Q. (2011). Removal of heavy metal ions from wastewaters: A review. Journal of Environmental Management, 92(3), 407-418. https://doi.org/10.1016/j.jenvman.2010.11.011

Hegazi, H. A. (2013). Removal of heavy metals from wastewater using agricultural and industrial wastes as adsorbents. HBRC Journal, 9(3), 276-282. https://doi.org/10.1016/j.hbrcj.2013.08.004

Kumari, S., \& Rath, P. K. (2014). Extraction and Characterization of Chitin and Chitosan from (Labeo rohit) Fish Scales. Procedia Materials Science, 6(Icmpc), 482-489. https://doi.org/10.1016/j.mspro.2014.07.062

Leflaive, X. (2009). Alternative Ways of Providing Water. OECD Reports.

Mathews, P. G. (2004). Design of Experiments with MINITAB - Paul G. Mathews - Google Books. (Paul O’Mara \& Annemieke Hytinen, Eds.) (I). Wisconsin: ASQ Quality Press. Retrieved from https://books.google.co.bw/books?hl=en\&lr=\&id=7RaoAgAAQBAJ\&oi=fnd\&pg=PR18\&dq=factorial + design + on + minitab\&ots=jnp_b5f09Z\&sig=HG_v3FXdsOZ74dLtCi6DGtgOXM8\&redir_esc $=\mathrm{y} \# \mathrm{v}=$ onepage\&q=factorialdesi gn on minitab\&f=false

Paull, B., \& Barron, L. (2004). Using ion chromatography to monitor haloacetic acids in drinking water: A review of current technologies. Journal of Chromatography A, 1046, 1-9. https://doi.org/10.1016/S0021-9673(04)01029-5

Pokhrel, D., \& Viraraghavan, T. (2006). Arsenic removal from aqueous solution by iron oxide-coated fungal biomass: A factorial design analysis. Water, Air, and Soil Pollution, 173(1-4), 195-208. https://doi.org/10.1007/s11270-005-9056-z

Ponnusami, V., Krithika, V., Madhuram, R., \& Srivastava, S. N. (2007). Biosorption of reactive dye using acid-treated rice husk: Factorial design analysis. Journal of Hazardous Materials, 142(1-2), 397-403. https://doi.org/10.1016/j.jhazmat.2006.08.040

Ponnusami, V., Madhuram, R., Krithika, V., \& Srivastava, S. N. (2008). Effects of process variables on kinetics of methylene blue sorption onto untreated guava (Psidium guajava) leaf powder: Statistical analysis. Chemical Engineering Journal, 140(1-3), 609-613. https://doi.org/10.1016/j.cej.2007.11.003

Prakash, O., Talat, M., Hasan, S. H., \& Pandey, R. K. (2008). Factorial design for the optimization of enzymatic detection of cadmium in aqueous solution using immobilized urease from vegetable waste. Bioresource Technology, 99(16), 7565-7572. https://doi.org/10.1016/j.biortech.2008.02.008

Rao, T. P., Kala, R., \& Daniel, S. (2006). Metal ion-imprinted polymers-Novel materials for selective recognition of inorganics. Analytica Chimica Acta. https://doi.org/10.1016/j.aca.2006.06.065

Renge, V. C., Khedkar, S. V, \& Pande, S. V. (2012). Removal of Heavy Metals From Wastewater Using Low Cost Adsorbents : a Review. Sci. Revs. Chem. Commun, 2(4), 580-584.

Vigneswaran, S., \& Sundaravadivel, M. (2004). RECYCLE AND REUSE OF DOMESTIC WASTEWATER. In Encyclopedia of Life Support Systems (EOLSS) (5th ed.). Encyclopedia of Life Support Systems (EOLSS).

\section{Copyrights}

Copyright for this article is retained by the author(s), with first publication rights granted to the journal.

This is an open-access article distributed under the terms and conditions of the Creative Commons Attribution license (http://creativecommons.org/licenses/by/4.0/). 